Proyecciones Journal of Mathematics

Vol. 37, N $^{\circ}$ 4, pp. 749-764, December 2018.

Universidad Católica del Norte

Antofagasta - Chile

\title{
New interpretation of elliptic Boundary value problems via invariant embedding approach and Yosida regularization
}

\author{
Nadra Bouarroudj \\ ENP Oran Maurice Audin, Algeria \\ Lekhmissi Belaib \\ University of Oran 1 Ahmed Ben Bella, Algeria \\ and \\ Bekkai Messirdi \\ Laboratory of Fundamental and App. Math. of Oran, Algeria \\ Received: March 2018. Accepted : July 2018
}

\begin{abstract}
The method of invariant embedding for the solutions of boundary value problems yields an equivalent formulation to the initial boundary value problems by a system of Riccati operator differential equations. A combined technique based on invariant embedding approach and Yosida regularization is proposed in this paper for solving abstract Riccati problems and Dirichlet problems for the Poisson equation over a circular domain. We exhibit, in polar coordinates, the associated Neumann to Dirichlet operator, somme concrete properties of this operator are given. It also comes that from the existence of a solution for the corresponding Riccati equation, the problem can be solved in appropriate Sobolev spaces.
\end{abstract}

Subjclass : 35J05, 35J25, 34A12.

Keywords : Elliptic boundary value problems, Invariant embedding method, Riccati operator differential equations, Yosida regularization, Neumann to Dirichlet operator. 


\section{Introduction}

For studying some questions of control theory, transport theory, quadratic eigenvalue and filtering problems, one faces the problem of solving Riccati operator differential equations over a given domain. This equation has been studied by several authors in different contexts, Lions [8] using a Galerkin method, Bensoussan [2] in the context of Kalman filtering and others. Henry and Ramos have proposed in [5] the technique of invariant embedding, introduced by Bellman in [1], for the resolution of Poisson's problem in a cylindrical domain. The problem is embedded in a family of similar problems defined on subcylinders limited by a moving boundary and they obtained a factorization in two uncoupled problems of parabolic type, see [9], [10], [11], [12], [13] and [6]. Here, this method is used on the same kind of equations on circular domains, which corresponds to certain problems of mathematical physics and in particular the problems of fluid mechanics and viscosity. Our work is based essentially on the techniques developed in [7] and [13].

Precisely, this work concerns the factorization of a second order elliptic boundary value problem defined in a bounded regular domain, in a system of uncoupled initial value problems, using the technique of invariant embedding. But because the induced Riccati operator differential equations consists of unbounded linear operators, a combined technique based on invariant embedding and Yosida regularization is used for solving Riccati problems over an open circular domain.

Consider the abstract differential Riccati equation in polar coordinates:

$$
\begin{aligned}
& \frac{\partial P}{\partial \rho}-\frac{1}{\rho^{2}} P \frac{\partial^{2}}{\partial \theta^{2}} P-\frac{1}{\rho} P-I=0, \\
& 0<b<\rho<a, \theta \in] 0,2 \pi[
\end{aligned}
$$

with the initial condition $P(a)=0$. This problem has been proposed in [7] for the study of Dirichlet problem for the Poisson equation defined over an open disk $\Omega$ of $\mathbf{R}^{2}$ :

$$
\left(\mathcal{P}_{1}\right)\left\{\begin{array}{l}
-\Delta u=f \quad \text { in } \Omega \\
\left.u\right|_{\Gamma_{a}}=0
\end{array}\right.
$$

where $\Gamma_{a}=\partial \Omega$ is the boundary of $\Omega$ and $P(x)$ is the Neumann to Dirichlet operator on $\Gamma_{x}$ the annulus centered at the origin with radius $\left.x \in\right] b, a[$. I is the identity operator. 
The well-posedness of (1.1) was proved in [7]. This result was essentially established via the technique of factorization of second order elliptic boundary value problems. The purpose of the factorization method is : proving the equivalence between a boundary value problem and system of uncoupled first order initial value problems. The way of obtaining it was the Galerkin method in [7] and is the Yosida regularization here.

In this paper we present a direct study of the abstract differential Riccati equations arising from the factorization of the Poisson equations in a circular domain using a Yosida regularization. Here the Neumann to Dirichlet operator satisfying the Riccati equation is determined via a Yosida regularization technique. In particular, due to the unboundedness of certain operators, the fixed point argument often used in similar situations does not work any more. Our approach is rigorous and to our knowledge, the established results are obtained in a new and interesting way, in particular they allow us to provide more information on systems of uncoupled first order initial value problems.

The paper is organized in the following way. In section 2 , we describe the factorization method by space invariant embedding of boundary value problems, $\left(\mathcal{P}_{0}\right)$ is reduced to a system of initial value problems using an operator satisfying a Riccati equation. The obtained Riccati equation is the analogous of the block $L U$ factorization of a block tridiagonal matrix.

In section 3, we introduce the Yosida regularization technique of the corresponding Riccati equation in polar coordinates. We construct a family of regularizing operators for the considered problem and we prove the convergence of this method.

The strong limit on regularization permits to define in section 4 the socalled Neumann to Dirichlet operator in polar coordinates. We give more concrete properties of this operator, it also comes that from the existence of a solution for the corresponding Riccati equation the problem $\left(\mathcal{P}_{0}\right)$ can be solved in appropriate Sobolev spaces.

\section{Factorization Method by space invariant embedding}

We use now a space invariant embedding technique along the radius of the annulus centered at the origin with inner radius $b$ and outer radius $a$, $0<b<a$, this technique is inspired in the temporal invariant embedding used by J.-L. Lions for the control of parabolic systems, it allows one to solve many boundary value problems in a simple way. The aim of the 
present section is to show how to factorize a second order elliptic boundary value problem in a circular domain, in a system of uncoupled initial value problems. This factorization can be viewed as an infinite dimensional extension of the block Gauss factorization for linear systems.

\subsection{Motivation}

We consider the boundary value problem on the interval $] 0,1[$ for the onedimensional Schrödinger equation:

$$
\left\{\begin{array}{l}
\left.-\alpha \frac{d^{2} u}{d \rho^{2}}+\beta u=f, \rho \in\right] 0,1[ \\
\frac{d u}{d \rho}(0)=u_{0} \text { and } u(1)=u_{1}
\end{array}\right.
$$

where $\alpha, \beta \in] 0,+\infty\left[, u_{0}, u_{1} \in \mathbf{R}\right.$ and $f \in L^{2}(] 0,1[)$.

We are motivated to factorize the Schrödinger operator $-\alpha \frac{d^{2}}{d \rho^{2}}+\beta$ with the constant potential $\beta$, we are then led to search two function $A(\rho)$ and $B(\rho)$ such that:

$$
-\alpha \frac{d^{2}}{d \rho^{2}}+\beta=-\alpha\left(\frac{d}{d \rho}+A(\rho)\right)\left(\frac{d}{d \rho}+B(\rho)\right) .
$$

So, for each $u \in C^{2}(] 0,1[)$, we obtain:

$$
-\alpha \frac{d^{2} u}{d \rho^{2}}+\beta u=-\alpha \frac{d^{2} u}{d \rho^{2}}-\alpha(A(\rho)+B(\rho)) \frac{d u}{d \rho}-\alpha\left(\frac{d B(\rho)}{d \rho}+A(\rho) B(\rho)\right) u .
$$

By identification, one must have:

$$
\left\{\begin{array}{l}
A(\rho)+B(\rho)=0 \\
-\alpha\left(\frac{d B(\rho)}{d \rho}+A(\rho) B(\rho)\right)=\beta
\end{array}\right.
$$

Thus, $B(\rho)=-A(\rho)$ and $\frac{d A}{d \rho}+A^{2}=\frac{\beta}{\alpha}$. If we set $A(0)=0$ and $\phi(\rho)=-\frac{d u}{d \rho}+A(\rho) u$, then $\phi(0)=-\frac{d u}{d \rho}(0)=-u_{0}$ and we deduce the following system of uncoupled equations:

$$
\begin{cases}\frac{d A}{d \rho}+A^{2}=\frac{\beta}{\alpha}, & A(0)=0 \\ \frac{d \phi}{d \rho}+A \phi=\frac{f}{\alpha}, & \phi(0)=-u_{0} \\ \frac{d u}{d \rho}-A \phi=-\phi & u(1)=u_{1}\end{cases}
$$

where the equation in $A$ is a Riccati equation. 


\subsection{Riccati equation associated with the problem $\left(\mathcal{P}_{0}\right)$}

Let us consider the Dirichlet problem $\left(\mathcal{P}_{0}\right)$ for the Poisson equation in the open annulus $] b, a[\times] 0,2 \pi[$ and introduce the polar coordinates $u(x, y)=$ $\hat{u}(\rho, \theta), x=\rho \cos \theta, y=\rho \sin \theta, \rho \in] b, a[$ and $\theta \in] 0,2 \pi[$, then:

$$
\left(\mathcal{P}_{1}\right)\left\{\begin{array}{l}
\left.-\frac{\partial^{2} \hat{u}}{\partial \rho^{2}}-\frac{1}{\rho} \frac{\partial \hat{u}}{\partial \rho}-\frac{1}{\rho^{2}} \frac{\partial^{2} \hat{u}}{\partial \theta^{2}}=f \text { in } \widehat{\Omega}=\right] b, a[\times] 0,2 \pi[ \\
\left.\hat{u}\right|_{\Gamma_{a}}=0 \\
\int_{\Gamma_{b}} \frac{\partial \hat{u}}{\partial \rho} d \Gamma_{b}=0 \\
\left.\hat{u}\right|_{\theta=0}=\left.\hat{u}\right|_{\theta=2 \pi} \\
\left.\frac{\partial \hat{u}}{\partial \theta}\right|_{\theta=0}=\left.\frac{\partial \hat{u}}{\partial \theta}\right|_{\theta=2 \pi}
\end{array}\right.
$$

where $\Gamma_{r}$ is the circle centred at the origin, with radius $r, r=a, b$. The choise of the boundary conditions on $\Gamma_{b}$ corresponds to a nul total flux.

We embed this problem in the family of similar problems defined by:

$$
\left(\mathcal{P}_{s, h}\right)\left\{\begin{array}{l}
\left.-\frac{\partial^{2} \hat{u}_{s}}{\partial \rho^{2}}-\frac{1}{\rho} \frac{\partial \hat{u}_{s}}{\partial \rho}-\frac{1}{\rho^{2}} \frac{\partial^{2} \hat{u}_{s}}{\partial \theta^{2}}=f \text { in } \widehat{\Omega} \backslash \widehat{\Omega}_{s}=\right] s, a[\times] 0,2 \pi[ \\
\left.\hat{u}_{s}\right|_{\Gamma_{a}}=0 \\
\left.\frac{\partial \hat{u}_{s}}{\partial \rho}\right|_{\Gamma_{s}}=h \\
\left.\hat{u}_{s}\right|_{\theta=0}=\left.\hat{u}_{s}\right|_{\theta=2 \pi}, \\
\left.\frac{\partial \hat{u}_{s}}{\partial \theta}\right|_{\theta=0}=\left.\frac{\partial \hat{u}_{s}}{\partial \theta}\right|_{\theta=2 \pi} .
\end{array}\right.
$$

By linearity of $\left(\mathcal{P}_{s, h}\right)$, the operator on $\Gamma_{s}:\left.h \mapsto \hat{u}_{s}\right|_{\Gamma_{s}}$ is affine, so:

$$
\left.\hat{u}_{s}\right|_{\Gamma_{s}}=P(s) h+r(s) .
$$

where $P(s)$ is the Neumann to Dirichlet map for the annulus $\widehat{\Omega} \backslash \widehat{\Omega}_{s}$. In fact $P(s)$ is the opposite of the Neumann-Dirichlet operator as the Neumann data is $-h$.

Furthermore, the solution $\hat{u}_{s}$ of $\left(\mathcal{P}_{s, h}\right)$ is given by:

$$
\hat{u}_{s}(\rho, \theta)=\left(\left.P(\rho) \frac{\partial \hat{u}_{s}}{\partial \rho}\right|_{\Gamma_{s}}\right)(\theta)+r(\rho, \theta)
$$


Formally derive the identity (2.1) with respect to $\rho$ and use the equation satisfied by $\hat{u}_{s}$, we obtain:

$$
\begin{aligned}
\frac{\partial \hat{u}_{s}}{\partial \rho} & =\frac{\partial P}{\partial \rho} \frac{\partial \hat{u}_{s}}{\partial \rho}+P \frac{\partial^{2} \hat{u}_{s}}{\partial \rho^{2}}+\frac{\partial r}{\partial \rho} \\
& =\frac{\partial P}{\partial \rho} \frac{\partial \hat{u}_{s}}{\partial \rho}+P\left(-f-\frac{1}{\rho^{2}} \frac{\partial^{2} \hat{u}_{s}}{\partial \theta^{2}}-\frac{1}{\rho} \frac{\partial \hat{u}_{s}}{\partial \rho}\right)+\frac{\partial r}{\partial \rho} \\
& =\frac{\partial P}{\partial \rho} \frac{\partial \hat{u}_{s}}{\partial \rho}-P f-P \frac{1}{\rho^{2}} \frac{\partial^{2}}{\partial \theta^{2}}\left(P \frac{\partial \hat{u}_{s}}{\partial \rho}+r\right)-P \frac{1}{\rho} \frac{\partial \hat{u}_{s}}{\partial \rho}+\frac{\partial r}{\partial \rho} \\
& =\left(\frac{\partial P}{\partial \rho}-P \frac{1}{\rho^{2}} \frac{\partial^{2}}{\partial \theta^{2}} P-P \frac{1}{\rho}\right) \frac{\partial \hat{u}_{s}}{\partial \rho}-P f-P \frac{1}{\rho^{2}} \frac{\partial^{2} r}{\partial \theta^{2}}+\frac{\partial r}{\partial \rho}
\end{aligned}
$$

Considering $\frac{\partial \hat{u}_{s}}{\partial \rho}$ arbitrary, one gets the following decoupled system:

$$
\begin{cases}\frac{\partial P}{\partial \rho}-\frac{1}{\rho^{2}} P \frac{\partial^{2}}{\partial \theta^{2}} P-\frac{1}{\rho} P-I=0, & P(a)=0 \\ -P f-P \frac{1}{\rho^{2}} \frac{\partial^{2} r}{\partial \theta^{2}}+\frac{\partial r}{\partial \rho}=0, & r(a)=0 \\ P \frac{\partial \hat{u}_{s}}{\partial \rho}-\hat{u}_{s}=-r . & \end{cases}
$$

where the initial conditions for $P$ and $r$ are directly obtained from (2.1) written at $\rho=a$. Note that the decoupled system of initial value problems in $P, r, u$ is equivalent to the boundary value problems as claimed in [7].

Let $P$ be the solution of the corresponding Riccati equation:

$$
\frac{\partial P}{\partial \rho}-\frac{1}{\rho^{2}} P \frac{\partial^{2}}{\partial \theta^{2}} P-\frac{1}{\rho} P-I=0, P(a)=0(2.2)
$$

we put

$$
P(\rho)=\rho Q(\rho)
$$

Then

$$
\rho \frac{\partial Q}{\partial \rho}-Q \frac{\partial^{2}}{\partial \theta^{2}} Q-I=0, Q(a)=0 .
$$

By making the change of variables $r=\ln \rho$, we get:

$$
\frac{\partial Q}{\partial r}-Q \frac{\partial^{2}}{\partial \theta^{2}} Q-I=0, Q(\ln a)=0
$$




\section{Yosida regularization technique}

Approximation methods play an important role in nonlinear analysis. A number of problems in variational analysis and in optimization theory give rise to nonsmooth functions with possibly infinite values defined on finite or infinite dimensional spaces. Our focus here is on the existence of solutions of boundary value problems via the Yosida regularization technique. This spectral method (see e.g. [4]) has been studied and has proven to be advantageous compared to other approaches. The key point to our analysis is to construct a family of regularizing operators for the considered problem and we prove the convergence of this method.

We denote by:

$$
\begin{gathered}
H_{\rho, P}^{k}(0,2 \pi)=\left\{v: v \in L^{2}(0,2 \pi), \frac{1}{\rho^{j}} \frac{\partial^{j} v}{\partial \theta^{j}} \in L^{2}(0,2 \pi),\right. \\
j=1, \ldots, k \text { and } v(0)=v(2 \pi)\}
\end{gathered}
$$

with $0<b<\rho<a, k=1,2$.

where $H=L^{2}(0,2 \pi)$ is the Hilbert space of Lebesgue square integrable functions on $(0,2 \pi)$ equipped with the natural inner product $(f, g)=$ $\int_{0}^{2 \pi} f(\theta) \overline{g(\theta)} d \theta$.

Let $A=-\frac{d^{2}}{d \theta^{2}}$ be the unbounded self-adjoint operator defined on $H$ with domain $D(A)=H_{\rho, P}^{2}(0,2 \pi)$ and let $A_{n}$ be its Yosida regularization given by:

$$
A_{n}=n I-n^{2}(n I+A)^{-1}, n \in \mathbf{N}^{*} .
$$

$A_{n}$ is well defined on $H$ since the spectrum of $A$ is embedded in $[0,+\infty[$. We also notice that:

$$
\lim _{n \rightarrow+\infty} A_{n} h=A h, \text { for all } h \in D(A) .
$$

Each $A_{n}$ is a positive operator on $H$ and so we can define the positive square root $A_{n}^{1 / 2}$ of $A_{n}$. Furthermore, $A_{n}^{1 / 2}$ is the infinitesimal generator of the strongly continuous one-parameter semigroup:

$$
w_{n}(r)=\exp \left(2 A_{n}^{1 / 2}(\ln a-r)\right), r \leq \ln a, n \in \mathbf{N}^{*} .
$$

Taking into account that $A_{n}^{1 / 2}$ is selfadjoint, then $w_{n}(r)$ is also selfadjoint for all $n \in \mathbf{N}^{*}$. We have: 


$$
\begin{aligned}
\left.\left(w_{n}(r)+I\right) h, h\right) & =\left(\exp \left(A_{n}^{1 / 2}(\ln a-r)\right) h, \exp \left(A_{n}^{1 / 2}(\ln a-r)\right) h\right)+(h, h) \\
& \geq\|h\|^{2}, \text { for all } h \in H
\end{aligned}
$$

$w_{n}(r)+I$ is continuous, then by Lax-Milgram theorem, we may conclude that it is invertible on $H$ with:

$$
\left.\| w_{n}(r)+I\right)^{-1} \|_{\mathcal{L}(H)} \leq 1 \text {, for all } r \leq \ln a \text { and } n \in \mathbf{N}^{*} .
$$

Let for every $n \in \mathbf{N}^{*}, P_{n}$ be the solution of the corresponding Riccati equation to $A_{n}$ :

$$
\frac{\partial P_{n}}{\partial \rho}+\frac{1}{\rho^{2}} P_{n} A_{n} P_{n}-\frac{1}{\rho} P_{n}-I=0, \quad P_{n}(a)=0 .
$$

and

$$
Q_{n}=\frac{P_{n}}{\rho}
$$

satisfying:

$$
\frac{\partial Q_{n}}{\partial r}+Q_{n} A_{n} Q_{n}-I=0, Q_{n}(\ln a)=0
$$

From [3], the equation (3.5) admits a solution given by:

$$
Q_{n}(r)=-A_{n}^{-1 / 2}\left(w_{n}(r)-I\right)\left(w_{n}(r)+I\right)^{-1}, n \in \mathbf{N}^{*} .
$$

Lemma 3.1. For $0<b<\rho<a, n \in \mathbf{N}^{*}$,

$$
P_{n}(\rho)=-\rho A_{n}^{-1 / 2}\left(w_{n}(\ln \rho)-I\right)\left(w_{n}(\ln \rho)+I\right)^{-1}
$$

is well defined and $P_{n}(\rho) \in \mathcal{L}(H)$ is negative self-adjoint operator on $H$. Moreover,

$$
P_{n} \in C^{1}([b, a] ; \mathcal{L}(H)) .
$$


Proof. For $\rho \in[b, a], P_{n}(\rho)$ is a product of self-adjoint, positive and bounded operators on $H$ which commute with each other and consequently we may conclude that $P_{n}(\rho)$ is self-adjoint, negative and bounded on $H$. For arbitrary $\rho \in[b, a]$ :

$$
\begin{aligned}
Q_{n}(\ln \rho)-Q_{n}\left(\ln \rho_{0}\right) & =-2 A_{n}^{-1 / 2}\left[\left(w_{n}\left(\ln \rho_{0}\right)+I\right)^{-1}-\left(w_{n}(\ln \rho)+I\right)^{-1}\right] \\
& =-2 A_{n}^{-1 / 2}\left(w_{n}(\ln \rho)-w_{n}\left(\ln \rho_{0}\right)\right)\left(w_{n}\left(\ln \rho_{0}\right)+I\right)^{-1}\left(w_{n}(\ln \rho)+I\right)^{-1}
\end{aligned}
$$

As $w_{n}(\ln \rho)=\exp \left(2 A_{n}^{\frac{1}{2}}(\ln a-\ln \rho)\right)$ is an uniformly continuous semigroup, we conclude that:

$$
\lim _{\rho \rightarrow \rho_{0}} \|\left(Q_{n}(\ln \rho)-Q_{n}\left(\ln \rho_{0}\right) \|=0\right.
$$

and so $P_{n} \in C([b, a] ; \mathcal{L}(H))$. We also have:

$$
\frac{Q_{n}(\ln \rho)-Q_{n}\left(\ln \rho_{0}\right)}{\rho-\rho_{0}}=-2 A_{n}^{-1 / 2} \frac{w_{n}(\ln \rho)-w_{n}\left(\ln \rho_{0}\right)}{\rho-\rho_{0}}\left(w_{n}\left(\ln \rho_{0}\right)+I\right)^{-1}\left(w_{n}(\ln \rho)+I\right)^{-1}
$$

and thus, for the same reason as before, $\frac{\partial P_{n}}{\partial \rho} \in C([b, a] ; \mathcal{L}(H))$. Consequently, $P_{n} \in C^{1}([b, a] ; \mathcal{L}(H))$.

Lemma 3.2. Let $0<b<\rho<a$. For each $h \in H$, there exists a constant $M(h) \geq 0$ such that:

$$
\left\|P_{n}(\rho) h\right\| \leq a M(h), \text { for all } \rho \in[b, a], n \in \mathbf{N}^{*} .
$$

Proof. Since by construction $A_{n}$ and $Q_{n}$ commute, then from the Riccati equation (3.5), we have:

$$
\left(A_{n}^{-1} \frac{\partial Q_{n}}{\partial r} h, h\right)+\left(Q_{n}^{2}(r) h, h\right)=\left(A_{n}^{-1} h, h\right), n \in \mathbf{N}^{*} .
$$

As $Q_{n}$ is a self-adjoint, then: $\left(A_{n}^{-1} \frac{\partial Q_{n}}{\partial r}(r) h, h\right)+\left\|Q_{n}(r) h\right\|^{2}=\left(A_{n}^{-1} h, h\right)$, for all $h \in H, r \leq \ln a, n \in \mathbf{N}^{*}$.

We may conclude from (3.6) that $A_{n}^{-1} \frac{\partial Q_{n}}{\partial r}$ is positive and so:

$$
\left\|Q_{n}(r) h\right\|^{2} \leq\left(A_{n}^{-1} h, h\right) \underset{n \longrightarrow \infty}{\longrightarrow}\left(A^{-1} h, h\right), \text { for all } h \in H, r \in[\ln b, \ln a]
$$


and consequently the sequence $\left(Q_{n}(r) h\right)_{n \in \mathbf{N}^{*}}$ is bounded in $H$, or for each $h \in H$, there exists a constant $M(h) \geq 0$ such that:

$$
\left\|Q_{n}(r) h\right\| \leq M(h), \text { for all } r \in[\ln b, \ln a], n \in \mathbf{N}^{*} .
$$

Returning to the variable $\rho$, we can write:

$$
\left\|Q_{n}(\ln \rho) h\right\| \leq M(h), \text { for all } \rho \in[b, a], n \in \mathbf{N}^{*} .
$$

Thus,

$$
\left\|P_{n}(\rho) h\right\|=\left\|\rho Q_{n}(\ln \rho) h\right\| \leq a M(h) \text {, for all } \rho \in[b, a], n \in \mathbf{N}^{*} .
$$

We verify in fact that the sequence $\left(P_{n}(\rho) h\right)_{n \in \mathbf{N}^{*}}$ is uniformly convergent in a sense that we explain in the following main theorem.

Theorem 3.3. For each $h \in H$, the sequence $\left(P_{n}(\rho) h\right)_{n \in \mathbf{N}^{*}}$ converges in $H$, the limit is attained uniformly for $\rho \in[b, a]$.

Proof. We have from (3.5) and by virtue of the commutativity of $A_{n}$ and $Q_{n}(r)$ :

$$
\begin{gathered}
A_{n} \frac{\partial Q_{n}(r)}{\partial r}+A_{n}^{2} Q_{n}^{2}(r)=A_{n} \\
\frac{\partial\left(A_{n} Q_{n}(r)\right)}{\partial r}+\left(A_{n} Q_{n}(r)\right)^{2}=A_{n}, n \in \mathbf{N}^{*} .
\end{gathered}
$$

Denote by $T_{n}(r)$ the operator $\left(-A_{n} Q_{n}(r)\right)$, then:

$$
-\frac{\partial T_{n}(r)}{\partial r}+T_{n}^{2}(r)=A_{n}, n \in \mathbf{N}^{*} .
$$

For each $r \in[\ln b, \ln a]$, consider the sequence $\left(T_{n}(r) h\right)_{n \in \mathbf{N}^{*}}$. Note that for each $r \leq \ln a$ and $n, m \in \mathbf{N}^{*}, T_{n}(r)$ and $T_{m}(r)$ commute with each other. So we have:

$$
\frac{\partial}{\partial r}\left(-T_{n}(r)+T_{m}(r)\right)+\left(T_{n}(r)+T_{m}(r)\right)\left(T_{n}(r)-T_{m}(r)\right)=\left(A_{n}-A_{m}\right) .
$$

By multiplying by $\left(T_{n}(r)-T_{m}(r)\right) h, h \in H$, it results that: 


$$
\begin{aligned}
& \left(\frac{\partial}{\partial r}\left(-T_{n}(r)+T_{m}(r)\right) h,\left(T_{n}(r)-T_{m}(r)\right) h\right)+ \\
& +\left(\left(T_{n}(r)+T_{m}(r)\right)\left(T_{n}(r)-T_{m}(r)\right) h,\left(T_{n}(r)-T_{m}(r)\right) h\right)= \\
& =\left(\left(A_{n}-A_{m}\right) h,\left(T_{n}(r)-T_{m}(r)\right) h\right) .
\end{aligned}
$$

As $\left(T_{n}(r)+T_{m}(r)\right)$ is a positive operator,

$$
\left(\left(T_{n}(r)+T_{m}(r)\right)\left(T_{n}(r)-T_{m}(r)\right) h,\left(T_{n}(r)-T_{m}(r)\right) h\right) \geq 0, \text { for all } h \in H
$$

we obtain:

$$
\begin{aligned}
& \left(-\frac{\partial}{\partial r}\left(T_{n}(r)-T_{m}(r)\right) h,\left(T_{n}(r)-T_{m}(r)\right) h\right) \\
& \leq\left(\left(A_{n}-A_{m}\right) h,\left(T_{n}(r)-T_{m}(r)\right) h\right)
\end{aligned}
$$

and

$$
\begin{aligned}
\left.-\frac{1}{2} \frac{\partial}{\partial r} \| T_{n}(r)-T_{m}(r)\right) h \|^{2} & \left.\left.=-\| T_{n}(r)-T_{m}(r)\right) h\left\|\frac{\partial}{\partial r}\right\| T_{n}(r)-T_{m}(r)\right) h \| \\
& \leq\left\|\left(A_{n}-A_{m}\right) h\right\|\left\|\left(T_{n}(r)-T_{m}(r)\right) h\right\|
\end{aligned}
$$

thus,

$$
-\frac{\partial}{\partial r}\left\|\left(T_{n}(r)-T_{m}(r)\right) h\right\| \leq\left\|\left(A_{n}-A_{m}\right) h\right\| .
$$

Now integrate this inequality between $r$ and $\ln a$. Using Lemma (3.2) and the condition $T_{n}(\ln a)=0$, we obtain for $r \in[\ln b, \ln a]$ and $h \in H$ :

$$
\begin{aligned}
\left\|\left(T_{n}(r)-T_{m}(r)\right) h\right\| & \leq(\ln a-r)\left\|\left(A_{n}-A_{m}\right) h\right\| \\
& \leq \ln a\left\|\left(A_{n}-A_{m}\right) h\right\| .
\end{aligned}
$$

Since $A_{n} h \underset{n \rightarrow \infty}{\longrightarrow} A h$, for all $h \in H$, and as $\left\|\left(A_{n}-A_{m}\right) h\right\|$ does not depend on $r$, we conclude that for each $h \in H,\left(T_{n}(r) h\right)_{n \in \mathbf{N}^{*}}$ is a Cauchy sequence in $H$, uniformly with respect to $r \in[\ln b, \ln a]$. Consequently, by the same reasoning on the equation for $Q_{n}$, we deduce that the sequence:

$P_{n}(r) h=-\rho A_{n}^{-1} T_{n}(r) h=-\rho\left(I+\frac{1}{n} A\right) A^{-1} T_{n}(r) h=-\rho A^{-1} T_{n}(r) h-\frac{\rho}{n} T_{n}(r) h$ is strongly convergent in $H$ uniformly with respect to $r \in[\ln b, \ln a]$. 


\section{Neumann to Dirichlet operator $P(\rho)$}

After passing to the limit when $n$ tends to infinity, we find the Neumann to Dirichlet operator $P(\rho)$ which is of interest for various kinds of problems as domain decomposition or the definition of transparent boundary conditions. Indeed, we can now define $P(\rho) h=\lim _{n \rightarrow+\infty} P_{n}(\rho) h$, as a $\mathcal{L}(H)$ operator, for each $\rho \in[b, a]$.

Theorem 4.1. The operator $Q=\frac{P(\rho)}{\rho}$ is a weak solution of Riccati equation (2.5) in the following sense:

$$
\frac{\partial}{\partial r}(Q h, g)+\left(\frac{\partial}{\partial \theta} Q h, \frac{\partial}{\partial \theta} Q g\right)=(h, g), \text { for all } h, g \in H,
$$

satisfying the condition $Q(\ln a)=0$.

Proof. We get from (3.5) and the self-adjointness of $A_{n}^{1 / 2}$ and $Q_{n}(r)$ :

$$
\left(\frac{\partial Q_{n}}{\partial r} h, g\right)+\left(A_{n}^{1 / 2} Q_{n}(r) h, A_{n}^{1 / 2} Q_{n}(r) g\right)=(h, g), \text { for all } h, g \in H .
$$

Let $\varphi \in \mathcal{D}(] \ln b, \ln a[)$, the space of infinitely-differentiable function of compact support in $] \ln b, \ln a[$. Multiplication of the previous differential equation with the appropriate function $r \varphi(r) \in \mathcal{D}(] \ln b, \ln a[)$ and integration of the resulting equation on $] \ln b, \ln a[$ :

$$
\int_{\ln b}^{\ln a}\left(\frac{\partial Q_{n}}{\partial r} h, g\right) r \varphi(r) d r+\int_{\ln b}^{\ln a}\left(A_{n}^{1 / 2} Q_{n}(r) h, A_{n}^{1 / 2} Q_{n}(r) g\right) r \varphi(r) d r
$$

$$
=\int_{\ln b}^{\ln a}(h, g) r \varphi(r) d r .
$$

From Fubini's theorem and integration by parts, we obtain since $Q_{n}(\ln a)=0$ and $Q_{n}(\ln b)=0$ :

$$
\int_{\ln b}^{\ln a}\left(\frac{\partial Q_{n}}{\partial r} h, g\right) r \varphi(r) d r=\int_{\ln b}^{\ln a}\left(\int_{0}^{2 \pi} \frac{\partial Q_{n}}{\partial r} h \bar{g} d \theta\right) r \varphi(r) d r
$$




$$
\begin{aligned}
& =\int_{0}^{2 \pi}\left(\int_{\ln b}^{\ln a} \frac{\partial Q_{n}}{\partial r} r \varphi(r) d r\right) h \bar{g} d \theta \\
& =\int_{0}^{2 \pi}(\underbrace{\left[Q_{n} r \varphi(r)\right]_{\ln b}^{\ln }}_{=0}-\int_{\ln b}^{\ln a} Q_{n} \frac{\partial}{\partial r}(r \varphi(r)) d r) h \bar{g} d \theta \\
& =-\int_{\ln b}^{\ln a}\left(Q_{n} f, g\right) r \varphi^{\prime}(r) d r-\int_{\ln b}^{\ln a}\left(Q_{n} f, g\right) \varphi(r) d r .
\end{aligned}
$$

For each $h, g$ fixed in $H$, we have:

$$
\lim _{n \rightarrow+\infty}\left(Q_{n}(r) h, g\right) \varphi^{\prime}(r)=(Q(r) h, g) \varphi^{\prime}(r), r \in[\ln b, \ln a]
$$

and from Lemma 3.2 :

$$
\left|\left(Q_{n}(r) h, g\right) \varphi^{\prime}(r)\right| \leq M(h) .\|g\|\left|\varphi^{\prime}(r)\right|, r \in[\ln b, \ln a], n \in \mathbf{N}^{*} .
$$

since each $A_{n}$ is bounded and $\left\|A_{n}^{1 / 2}\right\|=\left\|A_{n}\right\|^{1 / 2}$, for all $n \in \mathbf{N}^{*}$, if we put in the proof of theorem (3.3) $T_{n}(r)=A_{n}^{\frac{1}{2}} Q_{n}(r)$, we can demonstrate in the same way that $\lim _{n \rightarrow+\infty} A_{n}^{1 / 2} Q_{n}(r) h=A^{\frac{1}{2}} Q(r)$, then:

$$
\begin{gathered}
\lim _{n \rightarrow+\infty}\left(A_{n}^{1 / 2} Q_{n}(r) h, A_{n}^{1 / 2} Q_{n}(r) g\right) \varphi(r) \\
=\left(A^{1 / 2} Q(r) h, A^{1 / 2} Q(r) g\right) \varphi(r), r \in[\ln b, \ln a]
\end{gathered}
$$

and

$\left|\left(A_{n}^{1 / 2} Q_{n}(r) h, A_{n}^{1 / 2} Q_{n}(r) g\right) \varphi(r)\right| \leq M(h) M(g)|\varphi(r)|, r \in[\ln b, \ln a], n \in \mathbf{N}^{*}$.

By Lebesgue's theorem we can pass to the limit as $n \rightarrow \infty$ under the integral sign in (4.2), then (4.1) is satisfied in the sense of distributions on ] $\ln b, \ln a$ [ for all $h, g$ in $H$. Furthermore, since $Q_{n}(\ln a) h=0$, for all $h \in H$ and $n \in \mathbf{N}^{*}$, then $Q(\ln a)=0$.

Remark 4.2. In fact $P(\rho) h=\lim _{n \rightarrow+\infty} P_{n}(\rho) h \in H_{\rho, P}^{1}(0,2 \pi)$ for each $h \in$ $H$ and $\rho \in[b, a]$. Indeed, it is proved in Theorem 4.1 that $A^{1 / 2} Q$ is in $\mathcal{L}(H)$. Then it is sufficient to notice that $A^{1 / 2}$ defines an isomorphism between $H_{\rho, P}^{1}(0,2 \pi)$ and $H$ to get the result. 
Proposition 4.3. $\frac{\partial Q}{\partial r}$ is a positive operator on $H$ :

$$
\left(\frac{\partial Q}{\partial r} h, h\right) \geq 0, \text { for all } h \in H .
$$

Proof. From Theorem 4.1, we know that for all $f, g \in H$ and $n \in \mathbf{N}^{*}$ :

$$
\begin{aligned}
& \left(\frac{\partial}{\partial r} Q h, g\right)+\left(\frac{\partial}{\partial \theta} Q h, \frac{\partial}{\partial \theta} Q g\right)=(h, g) \\
& \left(\frac{\partial Q_{n}}{\partial r} h, g\right)+\left(A_{n}^{1 / 2} Q_{n}(r) h, A_{n}^{1 / 2} Q_{n}(r) g\right)=(h, g) .
\end{aligned}
$$

Taking into account that for each $h \in H, Q_{n}(r) h \underset{n \rightarrow+\infty}{\longrightarrow} Q(r) h$, uniformly with respect $r \in[\ln b, \ln a]$, we may conclude that:

$$
\left(\frac{\partial Q_{n}}{\partial r} h, h\right) \underset{n \rightarrow+\infty}{\longrightarrow}\left(\frac{\partial Q}{\partial r} h, h\right), \text { for all } h \in H .
$$

On the other hand, since $\frac{\partial Q_{n}}{\partial r} \geq 0$, then $\left(\frac{\partial Q}{\partial r} h, h\right) \geq 0$, for all $h \in H$.

Remark 4.4. The results previously established on the Neumann to Dirichlet operator provide an equivalent formulation of the problem $\left(\mathcal{P}_{0}\right)$ and solves this problem in an elegant way.

Acknowledgement: Authors are thankful to the honorable referee for valuable suggestions to improve the paper.

\section{References}

[1] Bellman, R., Dynamic Programming. Programming Princeton University Press, Princeton, (1957).

[2] Bensoussan, A., Filtrage optimal des systèmes lin éaires, Dunod, (1971).

[3] Bensoussan, A., Da Prato, G., Delfour, M. and Mitter, S., Representation and Control of Infinite Dimensional Systems, Birkh äuser, (2007).

[4] Bouarroudj, N., Belaib, L. and Messirdi, B., A Spectral Method for Fourth-Order Boundary Value-Problems, Mathematica, 60 (83), 2, pp. 111-118, (2018). 
[5] Henry, J. and Ramos, A.M., Factorization of second order elliptic boundary value problems by dynamic programming, Nonlinear Anal., 59, pp. 629-647, (2004).

[6] Henry, J. and Ramos, A.M., Factorization of boundary value problems using the invariant embedding method, ISTE press, October (2016).

[7] Henry, J., Louro, B. and Soares, MC., A factorization method for elliptic problems in a circular domain, C. R., Math., Acad. Sci. Paris, 339, pp. 175-180, (2004).

[8] Lions, J. L., Contrôle Optimal de Systèmes Gouvernés par des Équations aux Dérivées Partielles. Dunod, (1968).

[9] Louro, B., Henry, J. and Soares, MC., Factorization by invariant embedding of elliptic problems in a circular domain, System Modeling and Optimization, IFIP International Federation for Information Processing, 166, pp. 159-170, (2005).

[10] Louro, B., Henry, J. and Soares, MC., Factorization by Invariant Embedding of a Boundary Value Problem for the Laplace Operator, System Modeling and Optimization, IFIP Advances in Information and Communication Technology, 312, pp. 282-292, (2009).

[11] Louro, B., Henry, J. and Soares, MC., Factorization of linear elliptic boundary value problems in non cylindrical domains, C. R., Math., Acad. Sci. Paris, 349 (15-16), pp. 879-882, (2011).

[12] Louro, B., Henry, J. and Soares, MC., A factorization method for elliptic BVP, Proceedings of the 12th International Conference on Computational and Mathematical Methods in Science and Engineering, CMMSE, pp. 709-714, (2012), ISBN 978-84-615-5392-1.

[13] Soares, MC., Factorization by Invariant Embedding of Elliptic Problems: Circular and Star-shaped Domains, $\mathrm{PhD}$ thesis, Universidade Nova de Lisboa, (2006).

\section{Nadra Bouarroudj}

Department of Mathematics and informatics, ENP Oran Maurice Audin, Algeria

e-mail : nadra_belaib@yahoo.fr 


\section{Lekhmissi Belaib}

Department of Mathematics,

University of Oran 1 Ahmed Ben Bella,

Oran,

Algeria

e-mail : belaib_lekhmissi@yahoo.fr

and

Bekkai Messirdi

Laboratory of Fundamental and Applicable Mathematics of Oran (LMFAO)

Algeria

e-mail : bmessirdi@yahoo.fr 